\title{
The Application of Cuckoo Search Algorithm in the Path Planning of Logistics Vehicles with Time Windows
}

\author{
Lei Lei, a , Zhe Sun ${ }^{1}$, Chun Ying ${ }^{2}$, Shuhua Tan ${ }^{2}$ and Zhixin Sun ${ }^{1, b}$ \\ ${ }^{1}$ Nanjing University of Posts and Telecommunications, Nanjing 210003, China; \\ 2 The YuanTong Express, Shanghai 201705, China. \\ a17805123577@163.com, bsunzx@njupt.edu.cn
}

\begin{abstract}
The vehicle routing problems (VRP) in logistics distribution has far-reaching significance to logistics companies in reducing transportation costs and improving service quality and efficiency. A lot of research has been devoted to solve VRP. However, in practical applications, with the increase of constraints and optimization objectives, the dimension and difficulty of the problem increase exponentially, which will lead to a dramatic increase in the complexity of the algorithm and a decrease in performance. Therefore, a more efficient and simple algorithm is needed. Cuckoo Search Algorithm (CSA) is a new meta-heuristic algorithm proposed in 2009 with good global search capability and robustness, containing few parameters which has been validated to solve many optimization problems effectively. Therefore, this paper applies the CSA to solve the path planning problem of logistics vehicles, builds a vehicle routing problem with time windows (VRPTW) model, and validates that the CSA can effectively solve this optimization problem in an acceptable time by simulation.
\end{abstract}

Keywords: Cuckoo Search Algorithm; Vehicle Routing Problem with Time Windows; meta-heuristic algorithms; logistic distribution.

\section{Introduction}

With the continuous development of the economy, especially the rapid development of ecommerce in recent years, the scale of logistics distribution has expanded dramatically. Due to the expansion of logistics customers, increasingly complex transportation networks and unreasonable distribution routes, logistics distribution efficiency and service quality, as well as the utilization of resources, are seriously affected. Therefore, how to scientifically and reasonably find the optimal distribution route to reduce transportation costs and improve service quality is an important research content in the logistics industry.

The VRP (Vehicle Routing Problem) is one of the most basic issues in network optimization. It refers to designing a reasonable route arrangement for a given number of customers through a fleet [1]. With the continuous development and wide application of the VRP problem, VRP is extended to all kinds of variants. In logistic distribution, the logistics vehicle needs to serve customers within the given time window which is a service time interval determined by the earliest service time and the latest service time requested by the customer. This kind of VRP problem considering time constraint is called Vehicle Routing Problem with Time Windows (VRPTW).

In the VRPTW problem, the cost function includes transport cost and the time cost. Many researches have been done which proposed a variety of methods to solve VRPTW or enriched the VRPTW model by considering variety actual constraints. For instance, J Luo et al. proposed a novel hybrid shuffled frog leaping algorithm for VRPTW [2]. S Iqbal et al. proposed a new model for VRPTW and applied a hybrid methodology to solve it [3]. N Rincon-Garcia et al. obtained a reduction in the number of vehicles, travel distance and time [4]. F Hernandez et al. enrich the model with the multi-trip VRPTW [5]. To improve service quality and reduce the service time, Vitória Pureza et al. considered a number of extra deliverymen to be assigned to each route [6]. And Defu Zhang et al. introduced a new VRPTW with pallet loading constraints [7].

At present, the algorithms used to solve the VRPTW can be basically divided into three categories: precise algorithm, heuristic algorithm, and meta-heuristic algorithm. Among them, swarm intelligent algorithm shows better search performance and achieves satisfactory results. Such as Particle Swarm 
Optimization (PSO) [8], Ant Colony Optimization (ACO) [9], Genetic Algorithm (GA) [10], Firefly Algorithm (FA) [11]. However, in practical applications, especially in the logistics industry, many realistic constraints need to be considered, such as multiple distribution centers, vehicle load capacity, customer service time and customer priority, etc. The increase of dimension and difficulty of the problem leads to a dramatic increase in the complexity of the algorithm and a decrease in performance. Therefore, a more efficient and simpler algorithm is needed.

Yang and Deb proposed the Cuckoo Search Algorithm (CSA) in 2009[12]. In recent years, many scholars have devoted to improve the performance of CSA and exploring its applications which involve process parameter optimization, flow shop scheduling, multi-objective optimization $[13,14,15]$. Also, many studies have applied CSA to effectively solve CVRP and achieved good results $[16,17,18]$. In view of the special requirements of logistics and distribution for service time and cost, this paper plans to apply the CSA to the VRPTW model for logistics distribution and seek an effective and efficient solution method.

\section{The VRPTW Model and CSA}

\subsection{Mathematical Model of the VRPTW.}

Due to the fact that there is unavoidable deviation from the time windows in the actual distribution process, this paper fully considers the actual demand of the service quality and service time in the logistics industry and establish a VRPTW model for logistics distribution as follow.

We describe the VRPTW model for logistics distribution as one distribution center, N customers, $\mathrm{K}$ vehicles, and the maximum carrying capacity of each vehicle is $\mathrm{Q}$. If the service time deviates from the time window specified by the customer, the distribution center must pay a certain penalty. Each transport vehicle starts from the distribution center, and provides service to customers in order, and finally goes back to the distribution center. The symbols of model are described in Table 1.

Table 1. The symbolic description of the VRPSTW model

\begin{tabular}{|c|c|}
\hline $\mathrm{k}$ & The vehicle $\mathrm{k}$ \\
\hline $\mathrm{i}, \mathrm{j}$ & Customer $\mathrm{i}, \mathrm{j}$ \\
\hline $\mathrm{C}_{\mathrm{ij}}$ & Vehicle travel cost per unit distance between $\mathrm{i}$ and $\mathrm{j}$ \\
\hline $\mathrm{q}_{\mathrm{i}}$ & Demand of customer i \\
\hline$x_{i j k}\left\{\begin{array}{l}1 \\
0\end{array}\right.$ & $\begin{array}{c}\text { Vehicle } \mathrm{k} \text { travels from } \mathrm{i} \text { to } \mathrm{j} \\
\text { other }\end{array}$ \\
\hline$y_{i k}\left\{\begin{array}{l}1 \\
0\end{array}\right.$ & $\begin{array}{c}\text { The task of the customer i is completed by the vehicle } \mathrm{k} \\
\text { other }\end{array}$ \\
\hline $\mathrm{ET}_{i}$ & The earliest time for customer i to start service \\
\hline $\mathrm{LT}_{\mathrm{i}}$ & The latest service time required by customer $\mathrm{i}$ \\
\hline$S_{\mathrm{i}}$ & Time of vehicle k actually arrives at customer i \\
\hline$a_{i}$ & Penalty coefficient \\
\hline $\mathrm{P}_{\mathrm{i}}\left(\mathrm{S}_{\mathrm{i}}\right)$ & Penalty function \\
\hline
\end{tabular}

The penalty strategy. If the time when distribution vehicle I arrive at customer depot is earlier than $e_{i}$, the time penalty will be added to the transportation cost. If the time is later than $l_{i}$, the other vehicles will be dispatched.

$$
P_{i}\left(S_{i}\right)=\left\{\begin{array}{c}
a_{i}\left(E T_{i}-S_{i}\right), \quad S_{i}<E T_{i} \\
0, \quad E T_{i}<S_{i}<L_{i} \\
\infty, \quad S_{i}>L_{i}
\end{array}\right.
$$

According to the above description, the mathematical model of VRPSTW can be established as follows: 


$$
\operatorname{Min} \mathrm{Z}=\sum_{\mathrm{i}=0}^{\mathrm{N}} \sum_{\mathrm{j}=0}^{\mathrm{N}} \sum_{\mathrm{k}=1}^{\mathrm{k}} \mathrm{C}_{\mathrm{ij}} \cdot \mathrm{x}_{\mathrm{ijk}}+\sum_{\mathrm{i}-0}^{\mathrm{N}} \mathrm{P}_{\mathrm{i}}\left(\mathrm{S}_{\mathrm{i}}\right)
$$

Subject to

$$
\begin{gathered}
\sum_{\mathrm{i}=1}^{\mathrm{N}} \mathrm{q}_{\mathrm{i}} \mathrm{y}_{\mathrm{ik}} \leq \mathrm{Q}(\mathrm{k}=1,2 \ldots, \mathrm{K}) \\
\sum_{\mathrm{k}=1}^{\mathrm{K}} \mathrm{y}_{0 \mathrm{k}}=\mathrm{K} \\
\sum_{\mathrm{k}=1}^{\mathrm{K}} \mathrm{y}_{\mathrm{ik}}=1(\mathrm{i}=1,2, \ldots, \mathrm{N}) \\
\sum_{\mathrm{i}=0}^{\mathrm{N}} \mathrm{x}_{\mathrm{i} 0 \mathrm{k}}=1(\mathrm{k}=1,2, \ldots, \mathrm{K}) \\
\sum_{\mathrm{i}=0}^{\mathrm{N}} \mathrm{x}_{\mathrm{ijk}}=\mathrm{y}_{\mathrm{jk}}(\mathrm{j}=1,2, \ldots \mathrm{N} ; \mathrm{k}=1,2, \ldots, \mathrm{K}) \\
\sum_{\mathrm{i}=0}^{\mathrm{N}} \mathrm{x}_{\mathrm{ijk}}=\mathrm{y}_{\mathrm{ik}}(\mathrm{i}=1,2, \ldots, \mathrm{N} ; \mathrm{k}=1,2, \ldots, \mathrm{K})
\end{gathered}
$$

\subsection{Cuckoo Search Algorithm.}

CSA emulates the brood parasitism behavior of cuckoos and Levy flying behavior of birds. The formula for cuckoos finding the bird's nest path and updating its position is as follows:

$$
\mathrm{x}_{\mathrm{i}}^{\mathrm{t}+1}=\mathrm{x}_{\mathrm{i}}^{\mathrm{t}}+\alpha \oplus \mathrm{L}(\lambda)
$$

Where $x_{i}^{t}$ denotes the $i$-th solution of the $t$-th generation; $\alpha$ is a step factor that controls the range of random searches; $L(\lambda)$ is the Levy flight step length. The probability density function in the form of power function as:

$$
\mathrm{L}(\lambda) \sim \mu=t^{-\lambda}, 1<\lambda \leq 3
$$

The search path is composed of frequent short jumps and accidental long jump which makes CSA has a larger search space and more easily jump out of the local optimal. Besides, some inferior nests will be eliminated by a probability of discovery $\mathrm{P}_{a}$. The new nests are generated by random walk mechanism.

The main steps of CSA are described as follows:

1: Objective function $\mathrm{f}(\mathrm{X}), \mathrm{X}=\left(x_{1}, \ldots, x_{d}\right)^{T}$

2: Generate initial population of $\mathrm{n}$ host nests $X_{i}=(i=1, b, \ldots, n)$

3: while ( $\mathrm{t}<$ Max Generation) or (stop criterion) do

4: Get a cuckoo randomly by Levy flights

5: evaluate its quality fitness $F_{i}$

6: Choose $\alpha$ nest among $\mathrm{n}$ (say $\mathrm{j}$ ) randomly

7: $\quad$ if $F_{i}>F_{j}$ then replace $\mathrm{j}$ by the new solution;

8: A fraction $\mathrm{P}_{a}$ of worse nests are abandoned;

9: New nests are built;

10: Keep the best solutions (or nests with quality solutions);

11: Rank the solutions and find the current best

12: Post process results and visualization 


\section{Solving the VRPTW with CSA}

\subsection{The Description of Key Steps.}

Step 1: set up a model of the VRPTW in logistics distribution and stored in Customer data table, set up the number of demand points, give them a number from 0 to $\mathrm{N}$ (the distribution center mark 0 ), set the coordinates of the demand point and distribution center, the vehicle load, the speed, the time window (the earliest arrival time $\mathrm{e}_{i}$, the latest service time $\mathrm{l}_{i}$ ).

In the simulation experiment of this paper, we designed a distribution task includes 1 distribution centers and 19 customers. And the coordinates of the 20 points, the demands and time windows for each customer are given as follow.

Table 2. Customer data table

\begin{tabular}{|c|c|c|c|c|c|}
\hline \multirow{2}{*}{ Customer i } & \multirow{2}{*}{ Demand of customer i } & \multicolumn{2}{|c|}{ Customer position } & \multicolumn{2}{c|}{ Time Windows } \\
\cline { 2 - 6 } & 0 & $x_{i}$ & $\mathrm{y}_{i}$ & $\mathrm{e}_{i}$ & $\mathrm{l}_{i}$ \\
\hline 0 & 3.1 & 4.5 & 7.7 & 0 & 24 \\
\hline 1 & 0.8 & 5 & 10.1 & 3 & 7.2 \\
\hline 2 & $\ldots$ & 3.7 & 8.6 & 4 & 10 \\
\hline$\ldots$ & 1.5 & $\ldots$ & $\ldots$ & $\ldots$ & $\ldots$ \\
\hline 20 & & 8.7 & 2.1 & 4.3 & 7.8 \\
\hline
\end{tabular}

Step 2: calculate the linear distance array between the demand points $(0 \ldots \mathrm{N})$ and turn them into the symmetric matrix.

Step 3: Modeling the VRPSTW described above. The objective function is used to express the minimization of transportation cost $\mathrm{Z}=\sum_{\mathrm{i}=0}^{\mathrm{N}} \sum_{\mathrm{j}=0}^{\mathrm{N}} \sum_{\mathrm{k}=1}^{\mathrm{k}} \mathrm{C}_{\mathrm{ij}} \cdot \mathrm{x}_{\mathrm{ijk}}+\sum_{\mathrm{i}-0}^{\mathrm{N}} \mathrm{P}_{\mathrm{i}}\left(\mathrm{S}_{\mathrm{i}}\right)$ and it is also the fitness function of CSA.

Step 4: Call CSA to find the best delivery sequence.

Step 5: Output result.

\subsection{Simulation Results.}

The convergence result of CSA

In order to test the performance of CSA in solving the VRPSTW model proposed in this paper, we use ACO and PSO as the contrast algorithm to carry out simulation experiments. As FIG.1 shows, after the finite iteration, CSA can converge quickly to the lower objective function value (741.39) than ACO (756.17) and PSO (751.02). The fig.1 proves the effectiveness and superiority of CSA in solving the VRPSTW problem.

Optimal distribution path

As is shown in Fig.2, four vehicles are needed to complete the distribution task and the distribution sequence of each vehicle is arranged as follows.

The distribution path of the vehicle 1 is: $0 \rightarrow 1 \rightarrow 12 \rightarrow 20 \rightarrow 16 \rightarrow 2 \rightarrow 0$

The distribution path of the vehicle 2 is: $0 \rightarrow 19 \rightarrow 6 \rightarrow 9 \rightarrow 5 \rightarrow 0$

The distribution path of the vehicle 3 is: $0 \rightarrow 15 \rightarrow 10 \rightarrow 8 \rightarrow 3 \rightarrow 13 \rightarrow 18 \rightarrow 4 \rightarrow 0$

The distribution path of the vehicle 4 is: $0 \rightarrow 14 \rightarrow 7 \rightarrow 11 \rightarrow 17 \rightarrow 0$

Further the total transport distance can be acquired as 741.39. And assuming that the transport cost per unit $C_{i j}$ is 3.5 yuan, the total transport cost required for completing such a distribution task is 2594.89 yuan. 


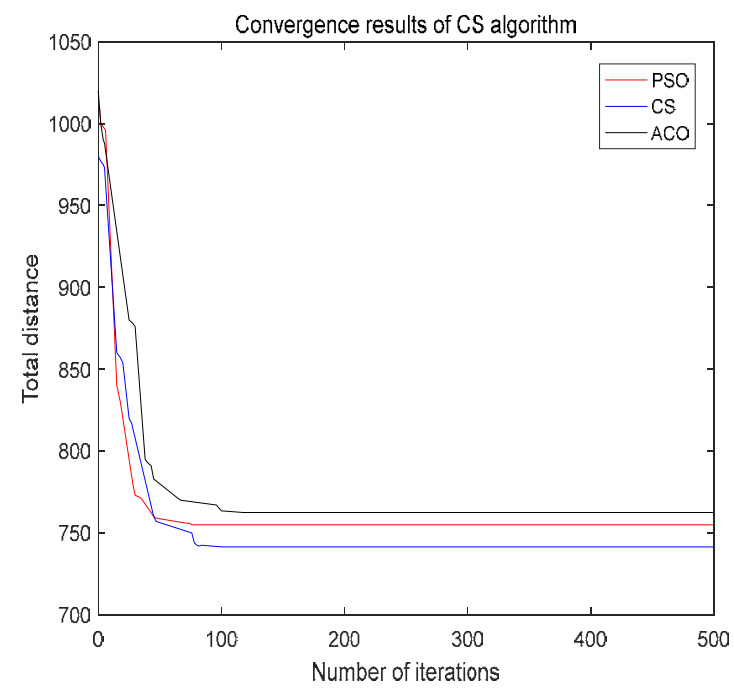

Fig. 1 The convergence graph of CSA

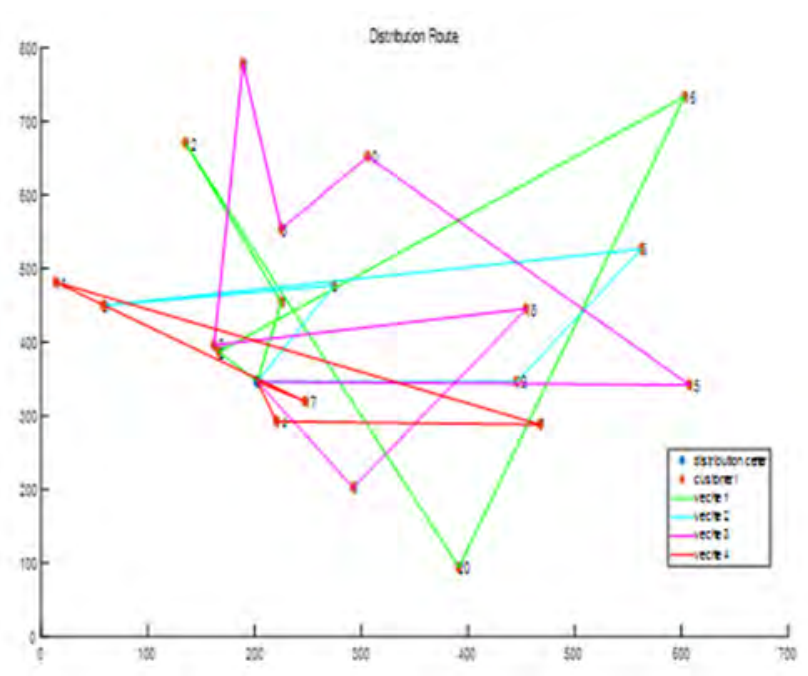

Fig. 2 The Distribution Route

\section{Summary}

Vehicle routing problem is one of main research directions of logistics distribution, and it is the key problem that logistics enterprises seek to reduce transportation cost, improve distribution efficiency and improve service quality. Considering of the actual demand of vehicle transportation in the logistics distribution, a VRPSTW model is established in this paper and adopts CSA to obtain the optimal distribution order. The simulation verified the effectiveness and rationality of the CSA to solve this VRPTW. In the future, when a large number of transportation vehicles are put into distribution and the constraints and objective functions are added, an efficient algorithm is especially needed. Therefore, this application is a meaningful attempt and exploration for the logistics vehicle routing planning.

\section{Acknowledgements}

This work is supported by the National Engineering Laboratory for Logistics Information Technology of YuanTong Express, the Natural Science Foundation of Jiangsu Province No. BK20160913 and No.BK20140883, the High Level Teacher Research Foundation of Nanjing University of Posts and Telecommunications No.NY2016021, The Chinese Natural Science Incubation Foundation of Nanjing University of Posts and Telecommunications No.NY217055, Jiangsu Postdoctoral Foundation No.1701016A, Natural Science Foundation of China No.61602259, No.61373135, No.61672299.

\section{References}

[1]. Prins C, Labadi N, Prodhon C, et al. Editorial: Metaheuristics for logistics and vehicle routing[J]. Computers \& Operations Research, 2010, 37(11):1833-1834.

[2]. Luo J, Li X, Chen M R, et al. A novel hybrid shuffled frog leaping algorithm for vehicle routing problem with time windows[J]. Information Sciences an International Journal, 2015, 316(C):266292.

[3]. Iqbal S, Kaykobad M, Rahman M S. Solving the multi-objective Vehicle Routing Problem with Soft Time Windows with the help of bees[J]. Swarm \& Evolutionary Computation, 2015, 24:5064.

[4]. Rincon-Garcia N, Waterson B, Cherrett T. A hybrid metaheuristic for the time-dependent vehicle routing problem with hard time windows[J]. 2017, 8(1):141-160. 
[5]. Hernandez F, Feillet D, Giroudeau R, et al. Branch-and-price algorithms for the solution of the multi-trip vehicle routing problem with time windows[J]. European Journal of Operational Research, 2016, 249(2):551-559.

[6]. Pureza V, Morabito R, Reimann M. Vehicle routing with multiple deliverymen: Modeling and heuristic approaches for the VRPTW[J]. European Journal of Operational Research, 2012, 218(3): 636-647.

[7]. Zhang D, Cai S, Ye F, et al. A hybrid algorithm for a vehicle routing problem with realistic constraints[J]. Information Sciences, 2017, 394: 167-182.

[8]. Ai T J, Kachitvichyanukul V. A Particle Swarm Optimisation for Vehicle Routing Problem with Time Windows[J]. International Journal of Operational Research, 2017, volume 6:519-537(19).

[9]. Lai M, Tong X. A metaheuristic method for vehicle routing problem based on improved ant colony optimization and Tabu search[J]. Journal of Industrial \& Management Optimization, 2017, $8(2): 469-484$.

[10]. Bouchra B, Btissam D, Mohammad C. A hybrid genetic algorithm for the static and dynamic vehicle routing problem with soft time windows[C]. International Conference on Logistics Operations Management. IEEE, 2016:1-9.

[11]. Aggarwal D, Kumar V, Girdhar A. Firefly Algorithm for the Vehicle Routing Problem with time windows[C]. IEEE International Conference on Intelligent Computing, Instrumentation \& Control Technologies. IEEE, 2017.

[12]. Yang X S, Deb S. Cuckoo Search via Levy Flights[J]. Mathematics, 2010:210 - 214.

[13]. Solihin M I, Zanil M F. Performance Comparison of Cuckoo Search and Differential Evolution Algorithm for Constrained Optimization[J]. 2016, 160(1):012108.

[14]. Hoang N D, Bui D T. A Novel Relevance Vector Machine Classifier with Cuckoo Search Optimization for Spatial Prediction of Landslides[J]. Journal of Computing in Civil Engineering, 2016, 30(5).

[15]. Nguyen T T, Vo D N. Modified cuckoo search algorithm for multiobjective short-term hydrothermal scheduling[J]. Swarm and Evolutionary Computation, 2017, 37: 73-89.

[16]. Teymourian E, Kayvanfar V, Komaki G H M, et al. Enhanced intelligent water drops and cuckoo search algorithms for solving the capacitated vehicle routing problem[J]. Information Sciences, 2016, 334: 354-378.

[17]. Berghida M, Boukra A. Resolving a Vehicle Routing Problem with Heterogeneous Fleet, Mixed Backhauls and Time Windows using Cuckoo Behavior Approach[J]. International Journal of Operational Research, 2015, 24(2).

[18]. Chen X, Wang J. A Novel Hybrid Cuckoo Search Algorithm for Optimizing Vehicle Routing Problem in Logistics Distribution System[J]. Journal of Computational \& Theoretical Nanoscience, 2016, 13(1):114-119. 